\title{
NEW MEANING OF EXACT CONTROLLABILITY OF LINEAR SYSTEMS IN HILBERT SPACES
}

\author{
Kangsheng Liu* \\ Department of Applied Mathematics \\ Zhejiang University, Hangzhou, 310027, China \\ Email: ksI@math.zju.edu.cn
}

David L. Russell

Department of Mathematics

Virginia Polytechnic Institute and State University

Blacksburg, Virginia, 24061, USA

\begin{abstract}
It is showed that under reasonable conditions, exact controllability of a linear system in a Hilbert space with bounded control implies time reversibility and spectral completeness of the system. The theory is applied to the beam and plate equations with boundary damping to establish their backward-time wellposedness and completeness of associated generalized eigenfunctions.
\end{abstract}

Key Words: exact controllability, $C_{0}$ group, generalized eigenfunctions, completeness, elastic system, damping

\section{INTRODUCTION}

There is an extensive literature on exact controllability for both abstract systems and concrete systems governed by partial differential equations ( cf. Russell [18], Pritchard and Zabczyk [17] Lions [6, 7], Komornik [5], Liu [8] and references cited therein). So far, exact controllability has been established almost in the systems which are reversible in time. Consider the wave and plate equations with boundary damping and distributed controls:

$$
\begin{cases}\ddot{w}-\Delta w=u & \text { in } \quad \Omega \times \mathbb{R}^{+} \\ \frac{\partial w}{\partial \nu}+\alpha(x) \dot{w}=0 & \text { on } \quad \partial \Omega \times \mathbb{R}^{+}\end{cases}
$$

*Supported partially by the National Key Project of China and by NSFC grant 19571073.

The original version of this chapter was revised: The copyright line was incorrect. This has been corrected. The Erratum to this chapter is available at DOI: 10.1007/978-0-387-35359-3_40 


$$
\left\{\begin{array}{l}
\ddot{w}+\Delta^{2} w=u \text { in } \Omega \times \mathbb{R}^{+} \\
y=0 \text { on } \partial \Omega \times \mathbb{R}^{+}, \quad \frac{\partial w}{\partial \nu}=0 \text { on } \Gamma_{0} \times \mathbb{R}^{+} \\
\Delta w=-\frac{\partial \dot{w}}{\partial \nu} \text { on } \Gamma_{1} \times \mathbb{R}^{+}
\end{array}\right.
$$

where $\Omega$ is a bounded open subset in $\mathbb{R}^{n}$ with the boundary $\partial \Omega$ smooth enough, $\nu$ is the unit normal vector of $\partial \Omega$ pointing towards the exterior of $\Omega, \alpha(x) \geq 0$ is a smooth function on $\partial \Omega, u \in L^{2}(\Omega \times \mathbb{R})$ is control, $\overline{\Gamma_{0}} \cup \overline{\Gamma_{1}}=\partial \Omega, \Gamma_{0} \cap \Gamma_{1}=\emptyset$. It has not been known whether the system (1.1) / (1.2) is exactly controllable in the finite energy state space, even in the one-dimensional case $(n=1)$. Majda [15] proved that the backward mixed problem of (1.1) is wellposed in the energy norm if and if $\alpha \equiv 0$. He [14] also showed that for certain $\alpha(x)$ the span of the generalized eigenfunctions associated with (1.1) has infinite codimension.

In this note, we announce the main results in Liu and Russell [12] (without the proofs) and apply them to establish backward-time wellposedness of (1.2), completeness of generalized eigenfunctions associated with (1.2), as well as lack of exact controllability of (1.1) when $\alpha\left(x_{0}\right) \neq 0$ for some $x_{0} \in \partial \Omega$.

\section{EXACT CONTROLLABILITY AND THE $C_{0}$ GROUP}

Let $H$ be a Hilbert space over the field $\mathbb{C}$ of complex numbers with the inner product $\langle\cdot, \cdot\rangle$ and the norm $\|\cdot\|$. Let $A$ generate a $C_{0}$ semigroup $e^{t A}$ on $H$. Consider the control system $(A, B)$ :

$$
y(u, t)=e^{t A} y_{0}+\int_{0}^{t} e^{(t-s) A} B u(s) d s
$$

where $y_{0} \in H, B \in \mathcal{L}(U ; H), U$ is another Hilbert space.

Definition 2.1 The system $(A, B)$ is said to be exactly controllable if for every $y_{0}, y_{1} \in H$ there exist $T>0, u(\cdot) \in L^{2}(0, T ; U)$ such that $y(u, 0)=y_{0}, y(u, T)=$ $y_{1}$. It is said to be reversible in time if $A$ generates a $C_{0}$ group.

Let us recall a fundamental result.

Proposition 2.1 (Zabczyk [20]) The following conditions are equivalent:

(1) The system $(A, B)$ is exactly controllable.

(2) There is a $T>0$ such that for every $y_{0}, y_{1} \in H$ there exists $u(\cdot) \in$ $L^{2}(0, T ; U)$ for which $y(u, 0)=y_{0}, y(u, T)=y_{1}$.

(3) (observability inequality) There exist $T, \delta>0$ such that

$$
\int_{0}^{T}\left\|B^{*} e^{t A^{*}} y\right\|_{U}^{2} d t \geq \delta\|y\|^{2} \quad \forall y \in H .
$$


In infinite-dimensional spaces, the class of systems $(A, B)$ which are exactly controllable is quite restricted. Pazy [16] proved that the observability inequality (2.2) with $U=H$ and $B=I$ holds if and only if for any $T>0$, there exist $\delta_{T}>0$ such that

$$
\left\|e^{t A^{*}} y\right\| \geq \delta_{T}\|y\| \quad \forall t \in[0, T], y \in H .
$$

As an immediate consequence of Pazy's result, the system $(A, B)$ is never exactly controllable if $e^{t A}$ is either compact or differentiable for $t \geq t_{0}>0$. Triggiani [19] proved that the system $(A, B)$ is never exactly controllable if $B$ is a compact operator. Louis and Wexler [13] proved that $(A, I)$ is exactly controllable if and only if there exists a $C_{0}$ semigroup $\hat{S}(t)$ on $H$ such that $e^{t A} \hat{S}(t)=I$ for all $t \geq 0$.

Let $L$ be a densely defined linear operator in $H$. We denote its defined domain, range, null space, adjoint operator, resolvent set, point spectrum by $D(L), R(L), \operatorname{Ker} L, L^{*}, \rho(L), \sigma_{p}(L)$, respectively. Let $\mathcal{L}(H)$ be the linear space of bounded linear operators on $H$. We define

$$
\begin{gathered}
\mathcal{S}=\left\{L \in \mathcal{L}(H) \mid L^{*}=L\right\}, \\
\mathcal{S}_{d}^{+}=\left\{L \in \mathcal{S} \mid \exists a>0 \text { such that }\langle L y, y\rangle \geq a\|y\|^{2} \forall y \in H\right\} . \\
\sigma_{p}^{*}(A)=\left\{\lambda \in \sigma_{p}(A) \mid R(\lambda I-A)=H\right\} .
\end{gathered}
$$

An operator $L \in \mathcal{L}(H)$ is said to be isometric if $\|L y\|=\|y\|$ for all $y \in H$; it is said to be unitary if $L^{*}=L^{-1}$. Now, we are in a position to present our results.

Theorem 1 (Liu and Russell [12]) The following statements are equivalent:

(a) The system $(A, I)$ is exactly controllable.

(b) There exist $P \in \mathcal{S}_{d}^{+}$., $Q \in \mathcal{S}$ such that $P e^{t\left(A^{*}+Q\right)} P^{-1}$ is a $C_{0}$ semigroup of isometric operators on $H$.

(c) There exist $P \in \mathcal{S}_{d}^{+}, Q \in \mathcal{S}$ such that

$$
e^{t A} P e^{t\left(A^{*}+Q\right)} P^{-1}=I \quad \forall t \geq 0 .
$$

Theorem 2 (Liu and Russell [12]) The following statements are equivalent:

(a) A generates a $C_{0}$ group on $H$.

(b) There exist $P \in \mathcal{S}_{d}^{+}, Q \in \mathcal{S}$ such that $P e^{t(A+Q)} P^{-1}$ is a $C_{0}$ group of unitary operators on $H$, i.e., $P(A+Q) P^{-1}$ is skew-adjoint.

(c) The system $(A, I)$ is exactly controllable and there exists a sequence $\left\{\lambda_{n}\right\}$ satisfying $\lambda_{n} \notin \sigma_{p}^{*}(A)$ and $\operatorname{Re} \lambda_{n} \rightarrow-\infty$. 
Liu [8] also proved that $e^{t A}$ can be embedded in a $C_{0}$ group on $H$ if and only if

(i) There exists $\sigma>0$ such that $\{\lambda \in \mathbb{C} \mid \operatorname{Re} \lambda \geq \sigma\} \subset \rho(-A)$.

(ii) There exists a nonincreasing function $\psi:[\sigma, \infty) \rightarrow(0, \infty)$ satisfying $\lim _{\tau \rightarrow+\infty} \psi(\tau)=0$, such that

$$
\left\|(\lambda+A)^{-1}\right\| \leq \psi(\operatorname{Re} \lambda) \quad \forall \operatorname{Re} \lambda \geq \sigma .
$$

Corollary 2.1 If the system $(A, B)$ with some bounded $B$ is exactly controllable and $A$ has compact resolvent, then $A$ generates a $C_{0}$ group on $H$.

Combination of Majda's result [15] and our corollary here yields the following:

Theorem 3 The system (1.1) is never exactly controllable if $\alpha\left(x_{0}\right) \neq 0$ for some $x_{0} \in \partial \Omega$.

Remark 2.1 From Theorem 2.2, if $(A, I)$ is exactly controllable but $A$ does not generate a $C_{0}$ group, then there exists $\sigma_{0} \in \mathbb{R}$ such that $\{\lambda \in \mathbb{C} \mid \operatorname{Re} \lambda \leq$ $\left.\sigma_{0}\right\} \subset \sigma_{p}^{*}(A)$. This case occurs when $e^{t A}$ is the semigroup of left translations on $L^{2}\left(\mathbb{R}^{+}\right)$(see Zabczyk [20]).

\section{THE COMPLETENESS OF GENERALIZED EIGENVECTORS}

Let $A$ be a closed linear operator defined in $H$. A nonzero vector $y$ is called a generalized eigenvector of $A$, corresponding to the eigenvalue $\lambda \in \sigma_{p}(A)$, if $(\lambda I-A)^{n} y=0$ for some positive integer $n$. Denote by $\operatorname{Sp}(A)$ the set of all finite linear combinations of the generalized eigenvectors. $A$ is said to have a complete system of generalized eigenvectors if $\operatorname{Sp}(A)$ is dense in $H$. The completeness of generalized eigenvectors is a desirable property in spectral analysis of linear operators. In the present section we will present this property for the infinitesimal generator of a $C_{0}$ group, by the theory in Gokhberg and Krein [3].

Let $\mathcal{M}_{\infty}$ be the set of all compact operators on $H$. For $L \in \mathcal{M}_{\infty}$, we have $0 \leq F \equiv\left(L^{*} L\right)^{\frac{1}{2}} \in \mathcal{M}_{\infty} \cap \mathcal{S}$. Following [3, §II.2], we enumerate the nonzero eigenvalues of $F$ in decreasing order, taking account of their multiplicities,

$$
s_{j}(L), \quad j=1,2, \cdots, r(F)=\operatorname{dim} R(F) .
$$

If $r(F)<\infty$, we put $s_{j}(L)=0$ for $j=r(F)+1, \cdots$. For $1 \leq p<\infty$, we define

$$
\mathcal{M}_{p}=\left\{L \in \mathcal{M}_{\infty} \mid \sum_{j=1}^{\infty} s_{j}^{p}(L)<+\infty\right\} .
$$

By the discussions in [3, Chap.III], we know that $\mathcal{M}_{p}$ is a two-sided ideal of the ring $\mathcal{L}(H)$. A compact operator is said to be of finite order if it is in $\mathcal{M}_{p}$ for some $1 \leq p<\infty$. 
Now, we are ready to present our completeness result.

Theorem 1 (Liu and Russell [12]) If $A$ is the infinitesimal generator of a $C_{0}$ group on a Hilbert space and $\left(\lambda_{0} I+A\right)^{-1}$ is a compact operator of finite order for some $\lambda_{0} \in \rho(A)$, then $A$ has a complete system of generalized eigenvectors.

We will apply our completeness theorem to the following variational evolution equation:

$$
\mathbf{c}(\ddot{w}(t), v)+\mathbf{b}(\dot{w}(t), v)+\mathbf{a}(w(t), v)=0, \quad \forall v \in V, \quad t>0 .
$$

A damped linear elastic system can be written in the form (3.1), where $\mathbf{a}(v, v)$ and $\mathbf{c}(v, v)$ are the strain energy and kinetic energy quadratic forms, respectively, $\operatorname{Re} \mathbf{b}(v, v)$ and $\operatorname{Im} \mathbf{b}(v, v)$ represent damping and gyroscopic forces, respectively. See K. Liu and Z. Liu [11], S. Chen, K. Liu and Z. Liu [2] for various properties of the $C_{0}$ semigroup associated with (3.1). We assume that

(H1) $V$ and $W$ are Hilbert spaces with inner products $\mathbf{a}(\cdot, \cdot)$ and $\mathbf{c}(\cdot, \cdot)$, respectively;

(H2) $V \hookrightarrow W$ is a compact and dense embedding;

Assumptions (H1) and (H2) imply that there exists a self-adjoint, positive definite operator $A_{0}$ in $W$ with compact resolvent such that ([4])

$$
D\left(A_{0}^{\frac{1}{2}}\right)=V, \quad \mathbf{a}(u, v)=\mathbf{c}\left(A_{0}^{\frac{1}{2}} u, A_{0}^{\frac{1}{2}} v\right), \quad \forall u, v \in V .
$$

It is clear that for every $0<\beta \leq 1, D\left(A_{0}^{\beta}\right)$ is also a Hilbert space with the inner product $\mathbf{c}\left(A^{\beta} \cdot A^{\beta} \cdot\right)$. We further assume that

(H3) $\mathbf{b}(\cdot, \cdot)$ is a continuous sesquilinear form on $D\left(A_{0}^{\theta}\right)$ for some $0 \leq \theta<\frac{1}{2}$.

By the Riesz representation theorem, assumption (H3) implies that there exists a compact operator $B_{1} \in \mathcal{L}\left(D\left(A_{0}^{\theta}\right)\right)$ such that

$$
\mathbf{b}(u, v)=\mathbf{c}\left(A_{0}^{\theta} B_{1} u, A_{0}^{\theta} v\right), \quad \forall u, v \in D\left(A_{0}^{\theta}\right) .
$$

Denote by $B=A_{0}^{\theta} B_{1}$. Then $B \in \mathcal{L}\left(D\left(A_{0}^{\theta}\right) ; W\right)$. Let $\mathcal{H}=V \times W$ with the naturally induced inner product

$$
\langle(u, v),(\hat{u}, \hat{v})\rangle_{\mathcal{H}}=\mathbf{a}(u, \hat{u})+\mathbf{c}(v, \hat{v})
$$

$\mathcal{H}$ may be regarded as the finite energy state space of the system (3.1). We define the operator $\mathcal{A}$ in $\mathcal{H}$ as follows:

$$
\left\{\begin{array}{l}
D(\mathcal{A})=\left\{(u, v) \mid u \in D\left(A_{0}^{1-\theta}\right), v \in V, A_{0}^{1-\theta} u+B v \in D\left(A_{0}^{\theta}\right)\right\} \\
\mathcal{A}(u, v)=\left(v,-A_{0}^{\theta}\left[A_{0}^{1-\theta} u+B v\right]\right) .
\end{array}\right.
$$

It is easy to check that

$$
\mathcal{A}^{-1}(u, v)=\left[\begin{array}{cc}
-A_{0}^{\theta-1} B & -A_{0}^{-1} \\
I & 0
\end{array}\right](u, v)
$$


for $(u, v):=(u, v)^{\tau} \in \mathcal{H}$. Obviously, $\mathcal{A}^{-1}$ is a compact operator in $\mathcal{L}(\mathcal{H})$. We will show the connection between the finite-order property of $\mathcal{A}^{-1}$ and the asymptotic distribution of eigenvalues of $A_{0}$.

Theorem 2 Let the assumptions (H1)-(H3) hold. Then the compact operator $\mathcal{A}^{-1}$ is of finite order if $A_{0}^{-1}$ is so.

Enumerating the eigenvalues of $A_{0}$ in increasing order, counting the multiple eigenvalues according to their multiplicity, we have

$$
0<\lambda_{1} \leq \lambda_{2} \cdots \lambda_{n} \leq \lambda_{n+1} \cdots .
$$

We know that $\lambda_{n} \rightarrow+\infty$ (since $\operatorname{dim} W=\infty$ ). We define

$$
N(\lambda)=\max \left\{j \mid \lambda_{j} \leq \lambda\right\} \quad \forall \lambda>0 .
$$

It is easy to see that $A_{0}^{-1}$ is finite order if

$$
N(\lambda)=\mathcal{O}\left(\lambda^{p}\right), \quad \lambda \rightarrow+\infty
$$

for some $p \geq 1$. We point out that the condition (3.9) always holds for a general self-adjoint elliptic operator $A_{0}$ on a bounded region (cf. Agmon [1] and the references therein).

\section{BACKWARD WELLPOSEDNESS AND SPECTRAL COMPLETENESS}

To write (1.2) with $u=0$ in the form (3.1), we can introduce

$$
\begin{aligned}
W & =L^{2}(\Omega), \quad V=\left\{v \in H^{2}(\Omega) \mid v=0 \text { on } \partial \Omega, \frac{\partial v}{\partial \nu}=0 \text { on } \Gamma_{0}\right\}, \\
\mathbf{c}(v, v) & =\int_{\Omega}|v|^{2} d x, \quad \mathbf{a}(v, v)=\int_{\Omega}|\Delta v|^{2} d x, \quad \mathbf{b}(v, v)=\int_{\Gamma_{1}}\left|\frac{\partial v}{\partial \nu}\right|^{2} d \sigma .
\end{aligned}
$$

Then, the assumptions (H1)-(H3) with $\theta=\frac{1-\epsilon}{2}$ for $0<\epsilon<\frac{1}{4}$ and the condition (3.9) are valid.

Theorem 1 If $\overline{\Gamma_{0}} \cap \overline{\Gamma_{1}}=\emptyset$, then $\mathcal{A}$ corresponding to (1.2) generates a $C_{0}$ group on $V \times W$ and has a complete system of generalized eigenvectors.

Sketch of the Proof. We know that $\mathcal{A}$ generates a $C_{0}$ semigroup $S(t)$ of contractions on $\mathcal{H}$ and $\mathcal{A}(w, v)=\left(v,-\Delta^{2} w\right)$,

$$
\begin{aligned}
& D(\mathcal{A})=\left\{(w, v) \mid w \in H^{4}(\Omega), w, v \in V, w=0 \text { on } \partial \Omega,\right. \\
& \left.\frac{\partial w}{\partial \nu}=0 \text { on } \Gamma_{0}, \Delta w=-\frac{\partial v}{\partial \nu} \text { on } \Gamma_{1}\right\} .
\end{aligned}
$$

For $(\xi, \eta) \in D(\mathcal{A})$, set $\left(w(t), w_{1}(t)\right)=S(t)(\xi, \eta)$. Then the function $w(\cdot)$ is in

$$
C\left([0, \infty) ; H^{4}(\Omega)\right) \cap C^{1}([0, \infty) ; V) \cap C^{2}([0, \infty) ; W) .
$$


It satisfies $\dot{w}(\cdot)=w_{1}(\cdot)$ and $(1.2)$ with $u=0$. Let

$$
E(t)=\frac{1}{2}\|S(t)(\xi, \eta)\|_{\mathcal{H}}^{2}=\frac{1}{2} \int_{\Omega}\left(|\Delta w|^{2}+|\dot{w}|^{2}\right) d x .
$$

We only need to prove the observability inequality

$$
\int_{0}^{T} E(t) d t \geq \delta E(0)
$$

for some $T, \delta>0$ and all real-valued $(\xi, \eta) \in D(\mathcal{A})$. Multiplying the first equation of $(1.2)(u=0)$ by $\dot{w}$ and integrating by parts, we obtain

$$
T E(0)-\int_{0}^{T} E(t) d t=\int_{0}^{T}(T-t) \int_{\Gamma_{1}}\left(\frac{\partial \dot{w}}{\partial \nu}\right)^{2} d \sigma d t
$$

We choose $h \in C^{1}\left(\bar{\Omega} ; \mathbb{R}^{n}\right)$ such that $h=0$ on $\Gamma_{0}$ and $h=\nu$ on $\Gamma_{1}$. Then we multiply the first equation of $(1.2)(u=0)$ by $(T-t) h \cdot \nabla w$ to get

$$
\begin{aligned}
& \int_{0}^{T}(T-t) \int_{\Gamma_{1}}\left[\left(\frac{\partial \dot{w}}{\partial \nu}\right)^{2}+\frac{1}{2} \operatorname{div} \frac{\partial \dot{w}}{\partial \nu} \frac{\partial w}{\partial \nu}\right] d \sigma d t \\
\leq & -\int_{\Omega} \eta h \cdot \nabla \xi d x+C_{T} \int_{0}^{T} E(t) d t .
\end{aligned}
$$

where $C_{T}$ is some positive number independent of $(\xi, \eta)$. By a compactness argument, we can now conclude that the observability inequality (4.4) is valid.

See Liu [10] for the details of proof and more examples dealing with backward wellposedness and spectral completeness of PDEs with boundary dissipation.

\section{References}

[1] S. Agmon, Asymptotic formulas with remainder estimates for eigenvalues of elliptic operators, Arch. Rational Mech. Anal., 28(1968), pp. 165-183.

[2] S. Chen, K. Liu and Z. Liu, Spectrum and stability of elastic systems with global or local Kelvin-Voigt damping, SIAM J. Appl. Math., to appear.

[3] I. C. Gokhberg and M. G. Krein, Introduction to the Theory of Linear Nonselfadjoint Operators, AMS, Providence, 1969.

[4] T. Kato, Perturbation Theory for Linear Operators, Springer-Verlag, New York, 1980.

[5] V. Komornik, Exact Controllability and Stabilization - The Multiplier Method, RAM, Masson, Paris, 1994.

[6] J.L. Lions, Exact controllability, stabilization and perturbations for distributed systems, SIAM Rev., 30(1988), pp.1-68. 
[7] -, Controlabilitè Exacte, Perturbations et Stabilisation de Systèmes Distribues, Tome 1, Collection RMA 8, Masson, Paris, 1988.

[8] K. Liu, Locally distributed control and damping for the conservative systems, SIAM J. Cont. Optim., 35(1997)5, pp. 1574-1590.

[9] K. Liu, A characterization of strongly continuous groups of linear operators on a Hilbert space, in submission.

[10] K. Liu, Backward wellposedness and spectral completeness of PDEs with boundary dissipation via exact observability, preprint.

[11] K. Liu and Z. Liu, Analyticity and differentiability of semigroups associated with elastic systems with damping and gyroscopic forces, J. Diff. Eqs., 141(1997)2, pp. 340-355.

[12] K. Liu and D. L. Russell, Exact controllability, time reversibility and spectral completeness of linear systems in Hilbert spaces, in submission.

[13] J. C. Louis and D. Wexler, On exact controllability in Hilbert spaces $J$. Diff. Eqns., 49(1983), pp. 258-269.

[14] A. Majda, Disappearing solutions for the dissipative wave equation, Indiana Univ. Math. J., 24(1975), pp. 1119-1133.

[15] A. Majda, The location of the spectrum for the dissipative acoustic operator, Indiana Univ. Math. J., 25(1976), pp. 973-987.

[16] A. Pazy, On the applicability of Lyapunov's theorem in Hilbert space, SIAM J. Math. Anal., 3(1972), pp. 291-294.

[17] A. J. Pritchard and J. Zabczyk, Stability and Stabilizability of infinite dimensional systems, SIAM Rev., 23(1981), pp.25-52.

[18] D. L. Russell, Controllability and stabilizability theory for linear PDE's: recent progress and open questions, SIAM Rev., 20(1978), pp.639-739.

[19] R. Triggiani, On the lack of exact controllability for mild solutions in Banach spaces, J. Math. Anal. Appl., 50(1975), pp. 438-446.

[20] J. Zabczyk, Remarks on the algebraic Riccati equation in Hilbert space, Appl. Math. Optim., 2(1976), pp. 251-258. 